mit Anleitung zur Technik und Methodik der Untersuchung. 1. und 2. Teil. Springer, Berlin (3. Teil Druck- und Verlagsanstalt, Zürich)

31. Butler HT (1927) An Illustrated Guide to the slit lamp. Oxford University Press, London

32. Kaschke M, Donnerhacke K-H, Rill MS (2014) Optical Devices in Ophthalmology and Optometry. Wiley-VCH, Weinheim

33. Meesmann A (1927) Die Mikroskopie des lebenden Auges an der Gullstrandschen Spaltlampe mit Atlas typischer Befunde. Urban \& Schwarzenberg, Berlin

\title{
Erratum
}

Ophthalmologe 2018 $1115: 892$

https://doi.org/10.1007/s00347-018-0776-z

Online publiziert: 20 . August 2018

(c) Springer Medizin Verlag GmbH, ein Teil von Springer Nature 2018

CrossMark

\section{H. Thomasen ${ }^{1}$ J. Schroeter ${ }^{2}$ - T. Reinhard ${ }^{3,5} \cdot$ B. Seitz ${ }^{4,5} \cdot$ K.-P. Steuhl' $\cdot$ D. Meller}

${ }^{1}$ Klinik für Augenheilkunde, Universität Duisburg-Essen, Essen, Deutschland

${ }^{2}$ Institut für Transfusionsmedizin, Universitätsgewebebank, Charité - Universitätsmedizin Berlin, Berlin Deutschland

${ }^{3}$ Universitäts-Augenklinik Freiburg, Freiburg, Deutschland

${ }^{4}$ Klinik für Augenheilkunde und Poliklinik, Universitätsklinikum des Saarlandes, UKS, Homburg/Saar, Deutschland

${ }^{5}$ Für: DOG-Sektion für Gewebetransplantation und Biotechnologie, München, Deutschland

${ }^{6}$ Klinik für Augenheilkunde, Universitätsklinikum Jena, Jena, Deutschland

\section{Erratum zu: Gute fachliche Praxis zur Gewinnung und Herstellung von kryokonservierter humaner Amnionmembran aus Spenderplazenta}

\section{Erratum zu:}

Ophthalmologe (2017)

https://doi.org/10.1007/s00347-017-

0626-4

Sehr geehrte Leserin, sehr geehrter Leser,

die zunächst veröffentlichte Onlineversion dieses Artikels war leider unvollständig, bei der in Kap. 8. Kontamination dargestellten Referenzliste fehlte der Punkt „sämtliche multiresistente Bakterien“.

Wir bitten, den korrigierten in dieser Ausgabe abgedruckten Beitrag zu berücksichtigen und den Fehler zu entschuldigen.

\section{Korrespondenzadresse}

Prof. Dr. D. Meller, FEBO

Klinik für Augenheilkunde, Universitätsklinikum Jena

Am Klinikum 1, 07747 Jena, Deutschland daniel.meller@med.uni-jena.de

Die Online-Version des Originalartikels ist unter https://doi.org/10.1007/s00347-017-0626-4zu finden. 